\title{
Sistemas de información enfocados en tecnologías de agricultura de precisión y aplicables a la caña de azúcar, una revisión
}

\author{
Óscar Arley Orozco* \\ Gonzalo Llano Ramírez
}

Recibido: 28/07/2015 - Aceptado: 11/12/2015

DOI: 10.22395/rium.v15n28a6

\begin{abstract}
Resumen
Los cultivos de caña de azúcar son una de las principales actividades económicas en Colombia; por ende, son esenciales para el desarrollo agrícola del país. Además, las tecnologías de la información y las comunicaciones (TIC) se han empezado a utilizar e implementar en todo el ciclo de vida del cultivo. Consecuentemente, las TIC son importantes al momento de definir sistemas basados en Agricultura de Precisión (AP), capaces de incrementar el rendimiento del cultivo y optimizar el uso de recursos económicos como fertilizantes, agua y pesticidas, entre otras funciones. Este artículo presenta una revisión acerca de sistemas de información basados en AP y aplicables a cultivos de caña de azúcar, haciendo énfasis en las tecnologías utilizadas, la gestión de datos y sus arquitecturas. Además, se presenta la propuesta de los autores: un sistema de información integral de tres capas basado en AP, capaz de facilitar la optimización en distintas etapas del ciclo de vida de la caña de azúcar. El artículo concluye describiendo el trabajo futuro y el desarrollo de la implementación del sistema propuesto.
\end{abstract}

Palabras clave: agricultura de precisión, caña de azúcar, rendimiento del cultivo, sistema de información, sistema de soporte a decisiones

Ingeniero en Electrónica y Telecomunicaciones, Universidad del Cauca - Estudiante de Maestría en Informática y Telecomunicaciones y miembro del Grupo de Investigación en Informática y Telecomunicaciones i2t - Universidad Icesi - Calle 18 \# 122-135, Cali, Valle - (2) 5552334 Ext 8529 - oaorozco@icesi.edu.co

** Doctor Ingeniero de Telecomunicaciones, Universidad Politécnica de Valencia, España - Director de la Maestría en Informática y Telecomunicaciones, investigador y profesor asociado - Universidad Icesi - Calle 18 \# 122-135, Cali, Valle - (2) 5552334 Ext 8395 - gllano@icesi.edu.co 


\title{
Information systems focused on precision agricultural technologies applicable to sugar cane, a review
}

\begin{abstract}
Crops of sugar cane are one of the main economic activities in Colombia. Hence, this kind of crops is essential for the agricultural development of the country. Additionally, information and communication technologies (ICTs) are currently used and implemented throughout the entire life of the crop. Therefore, ICTs are important at the time of defining PA-based systems, capable of increasing crop efficiency and optimizing use of economic resources as fertilizers, water, and pesticides, among other functions. This article presents a review about the PA-based information systems applicable to sugar cane crops and making emphasis on technologies used, data management, and their architectures. Besides, the article makes a proposal of authors: a AP-based three-layer integral information system capable of facilitating optimization in different life stages of the sugar cane. This article concludes by describing the future work and the implementation of the system proposed.
\end{abstract}

Key words: Precision agriculture; sugar cane; crop efficiency; information system; decision support system. 


\section{INTRODUCCIÓN}

La agricultura es una labor que acompaña al hombre hace miles de años, y sus procesos han evolucionado con el transcurso del tiempo. Según la FAO, un tercio de la población global deriva su sustento de la agricultura, y en economías emergentes esta puede representar hasta el $30 \%$ del PIB [1]. En Colombia, el Plan Nacional de Desarrollo plantea como estrategia transversal la transformación del campo y crecimiento verde para fortalecer la competitividad del sector agrícola buscando modernizar procesos y proyectos. Por tanto, dicho sector requiere la integración de diversos actores para alcanzar la modernización. En años recientes, empresas privadas y públicas pertenecientes al sector industrial, agrícola y de las TIC han unido esfuerzos para proyectar soluciones en el marco de la Agricultura de Precisión (AP), cuyo propósito es mejorar el rendimiento de cultivos, optimizar el uso de recursos, disminuir el impacto ambiental y facilitar la toma de decisiones estratégicas y económicas [2].

Por lo anterior, la AP presenta soluciones de modernización agrícola, mediante la integración de las TIC en los procesos de preparación, cultivo, recolección y producción de los cultivos para permitir desarrollar y articular procesos agrícolas más eficientes, confiables, modernos y seguros. El objetivo general de la agricultura de precisión es integrar la gestión espacial y temporal de la producción a través de Sistemas de Posicionamiento Global (GPS, Global Positioning Systems), Sistemas de Información Geográfica (SIG), tecnologías de sensores y mapas de terrenos. Lo anterior con el fin de aumentar la rentabilidad y productividad de los agricultores mediante la generación de mapas de productividad en función de las condiciones agroclimáticas del terreno [3].

La adopción de dichos elementos y tecnologías varía en función del cultivo, su ubicación geográfica y sus implicaciones derivadas (clima, humedad, tipo de suelo, etc.), además de las necesidades particulares de las empresas y las políticas gubernamentales. Considerando que en Colombia casi la totalidad de los cultivos de caña de azúcar están ubicados en el valle geográfico del río Cauca, el desarrollo industrial del cultivo está centrado en dicho sector, en donde se ubican ingenios, asociaciones y entidades relacionadas. El propósito de este artículo es realizar un estudio acerca de sistemas de información para AP, junto con sus principales características y posibles requerimientos de los actores presentes en el ciclo de vida del cultivo. En vista de lo anterior, se propone un sistema enfocado en la industria azucarera colombiana, orientado a proponer soluciones a dichas necesidades y requerimientos.

El resto del documento se estructura de la siguiente manera: la sección 1 presenta definiciones, elementos generales y desafíos de la agricultura de precisión; en la sección 2 se resalta el trabajo enfocado hacia sistemas de información basados en AP y otras propuestas relevantes; la sección 3 introduce detalles del sistema propuesto, su 
arquitectura y elementos, y describe el trabajo futuro de esta investigación; finalmente, la sección 4 concluye el documento.

\section{AGRICULTURA DE PRECISIÓN}

La agricultura de precisión surgió en Estados Unidos como un ciclo de realimentación anual donde se obtenían resultados relacionados con aplicación de insumos en función de las necesidades específicas del terreno. Con el paso del tiempo, se empezaron a utilizar las TIC para mejorar el manejo de suelos y cultivos; como resultado, las aplicaciones de AP se extendieron y adaptaron a diferentes labranzas, productos y países [4]. La AP se define como un conjunto de procedimientos y procesos que buscan optimizar espacial y temporalmente el ciclo de vida de diferentes cultivos a través de tecnologías, elementos y estudios de manera amigable con el medio ambiente.

Existe un consenso general acerca de las tecnologías y elementos que se utilizan en la agricultura de precisión, los cuales se encuentran abarcados por la geomática o disciplina de reunir, analizar, interpretar, distribuir y utilizar información geográfica. Dichas tecnologías y elementos se describen a continuación:

- Sistemas de posicionamiento: claves para el control de tráfico agrícola, puesto que proveen datos en tiempo real de su ubicación, facilitan así su gestión y control, y permiten la implementación de rutas óptimas. Dentro de estos sistemas se destacan GPS, GLONASS, Galileo y BeiDou.

- Tecnologías de tasa variable (VRT, Variable Rate Technologies): hacen referencia a elementos de aplicación de fertilizantes, pesticidas, herbicidas, agua y suplementos necesarios para el cultivo de manera autónoma y dependiente del lugar. Puesto que, dependiendo del sector o área específica del terreno cultivado, la necesidad de requerimientos puede ser diferente, las VRT juegan un papel importante en la optimización de recursos.

- Sensores remotos: encargados de capturar datos del cultivo, suelo, humedad, precipitaciones, entre otros, con ayuda de tecnologías inalámbricas como Wi-Fiß, Bluetooth ${ }^{\circledR}$ y redes celulares. Dicha captura se realiza con el objetivo de adquirir información acerca de la condición del suelo, crecimiento de las plantas, infestación de plagas, niveles de agua y fertilizantes.

- Sistemas de recomendación aplicados a cosechas: realizan predicciones basados en datos de entrada, ayudándose de algoritmos de machine learning. Específicamente para el tópico agrícola, dichos sistemas presentan mapas de rendimiento y mapas 
de productividad de los cultivos basados en información de cosechas pasadas, lo que facilita y optimiza la gestión de los cultivos.

- Aeronaves pilotadas remotamente (RPA, Remotely Piloted Aircraft): también denominados drones, ofrecen soluciones novedosas y económicas en el ámbito de obtención de imágenes en zonas de difícil acceso, estimación de variables agroclimáticas y monitorización remota de cultivos. Aunque pueden llegar a requerirse permisos y licencias gubernamentales que varían de país a país para su utilización, su uso se ha incrementado en los últimos años por la reducción en su precio y la facilidad para adquirirlos.

- Sistemas de soporte a decisiones (DSS, Decision Support Systems): hacen referencia a un conjunto de sistemas de información que complementa los anteriores componentes al facilitar la toma de decisiones por parte del personal relacionado con AP. Los DSS reciben información de sistemas de posicionamiento, de sensores remotos, de elementos que utilizan VRT y de drones, para su posterior procesamiento y despliegue al personal relacionado con el cultivo. Cabe destacar que el sistema propuesto recae en esta clasificación.

\subsection{Esquema general de AP}

Los elementos descritos anteriormente conforman el dominio para la aplicación de AP en un cultivo. La transferencia, almacenamiento y procesamiento de datos facilitan la toma de decisiones de tipo económico, ambiental y logístico. La figura 1 [5] muestra un esquema general del proceso de AP en donde pueden utilizarse, además de GPS, los demás elementos descritos previamente. De la figura se observa que el esquema es cíclico, por lo que los procesos y las decisiones tomadas en etapas previas se tienen en cuenta para etapas futuras. 


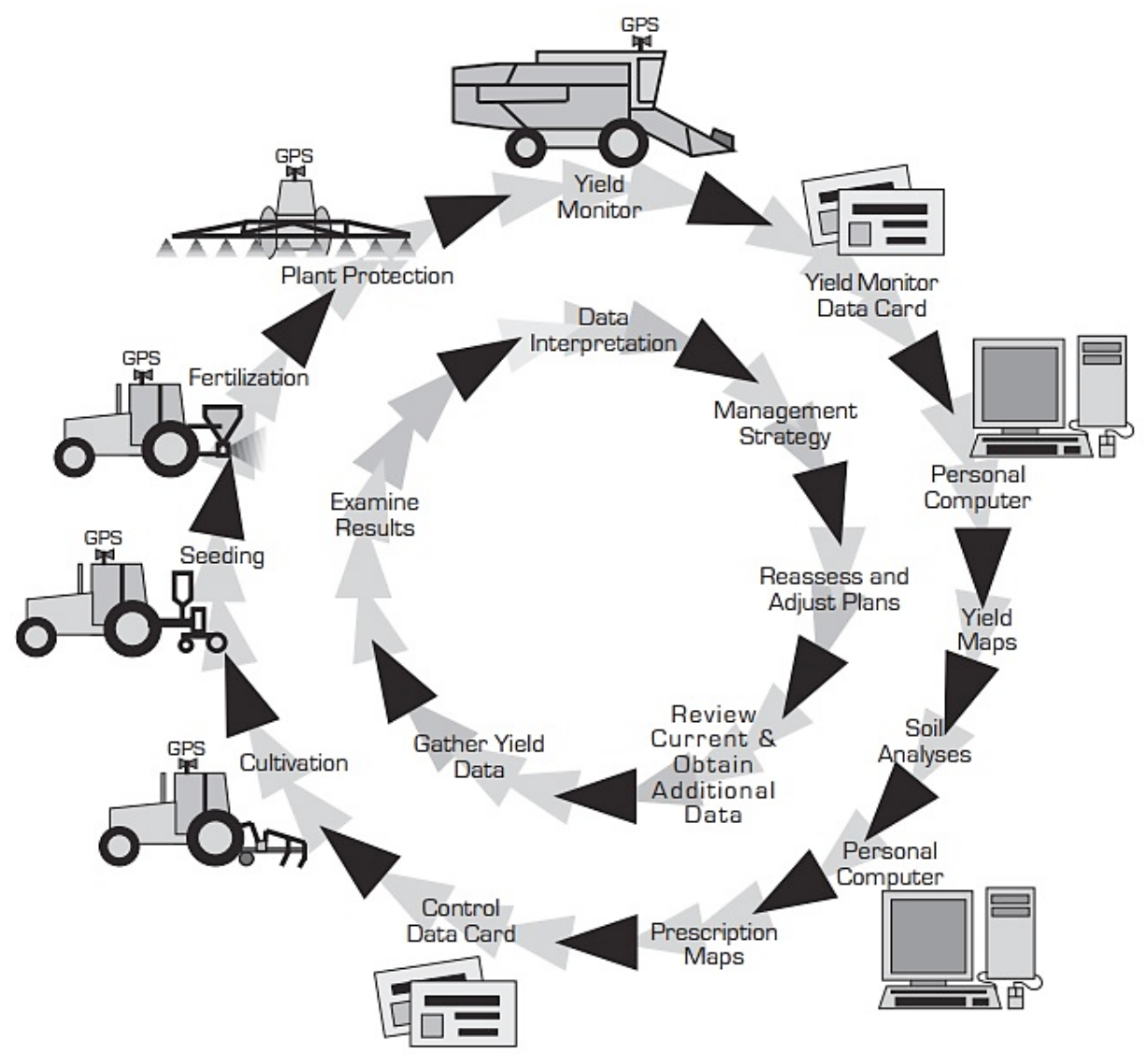

Figura 1. Esquema general del proceso de agricultura de precisión aplicado a un cultivo. Fuente: [5]

Si bien es cierto que el esquema de la figura 1 es general, para cada caso particular se adecuan los procedimientos y acciones a realizar. Como ejemplo, en Colombia la implementación de tecnologías y procedimientos de AP está aún en fases iniciales; pero se deben tener en cuenta elementos propios de la geografía, el cultivo, el suelo y el clima de la región en estudio; esto para lograr una adecuada identificación del cultivo y sus características intrínsecas, facilitando así la implementación de procesos de agricultura de precisión.

\subsection{Desafíos de la agricultura de precisión}

Dada la naturaleza de la AP, los desafíos que presenta se consideran como exigencias adicionales a su adecuada implementación. La importancia de resaltarlos recae en conocer qué impedimentos puede llegar a afrontar dicha implementación en el entorno 
colombiano. Los desafíos más importantes y estrategias para enfrentarlos se destacan a continuación $[3,6-8]$ :

- La inversión inicial para implementar tecnologías y procedimientos de AP es considerable y el retorno sobre la inversión (ROI, Return of Investment) requiere tiempo. Por tanto, para los agricultores con poca capacidad adquisitiva es complejo planear y ejecutar dichos proyectos. Para mitigar este desafío, es preciso que los gobiernos ejecuten políticas para ayudar a los agricultores a mejorar sus procesos productivos. Algunas políticas pueden ser: facilidad en la adjudicación de créditos para elementos de AP, junto con acompañamiento y asesoría en la utilización y mantenimiento, entre otros.

- La complejidad de las nuevas herramientas y tecnologías implica un mayor grado de aprendizaje de habilidades para el correcto manejo de estas, lo que puede variar de persona a persona, pudiendo llegar a afectar así el adecuado despliegue de AP. Para esto, se requiere de capacitación e información al personal que utilizará los elementos de AP para mitigar los posibles riesgos del cambio tecnológico.

- En economías emergentes predomina la agricultura tradicional; de ahí que con la implementación de AP se podría inferir erróneamente que se reemplazaría la mano de obra, lo que generaría problemas sociales. Para que esto no suceda, es necesario realizar campañas de sensibilización e información acerca de los potenciales beneficios de la agricultura de precisión y recalcar que esta no reemplazará a dicha mano de obra.

- No existen estándares o reglas de decisión globales hacia la implementación, uso y adopción de tecnologías de AP para los productores, lo que dificulta los tiempos de implementación. Esto es particularmente complicado, puesto que los cultivos y sus requerimientos dependen considerablemente de la ubicación, el clima, el tipo de suelo, entre otras variables. Por esto, estandarizar procedimientos y técnicas se convierte en una tarea minuciosa y laboriosa; la estrategia para la mitigación de este desafío recaería más en estudiar casos ya implementados de tecnologías de AP y analizar cómo adaptar dichas implementaciones a entornos locales.

\section{MÉTODO DE INVESTIGACIÓN}

Con el fin de establecer un punto de partida alrededor del conocimiento generado en el campo de sistemas de información basados en tecnologías de AP, se siguió la propuesta de Kitchenham [9] para la elaboración de la revisión de literatura. Aunque esta 
propuesta está enfocada a revisiones en ingeniería de software, es adaptable a otras temáticas. El procedimiento para realizar la revisión consiste en cinco pasos: definir preguntas de investigación, realizar la búsqueda literaria, seleccionar estudios, clasificar artículos y extraer y realizar la agregación de datos. En las siguientes secciones se describen estos cinco pasos.

\subsection{Preguntas de investigación}

El objetivo principal de este estudio es inspeccionar los sistemas de información basados en tecnologías de AP y cuál es la tendencia en este tópico. Con el fin de obtener un conocimiento más detallado y una visión integral del tema, se plantean las siguientes preguntas de investigación:

- ¿Qué temas interesan a la comunidad científica respecto a sistemas de información basados en AP dentro de un marco temporal reciente (últimos 10 años)?

- ¿Qué características presentan estos sistemas en relación a su arquitectura, funcionamiento y aplicabilidad a diversos cultivos?

- ¿Cuáles son las técnicas, procedimientos y tecnologías que aún no han sido exploradas ni utilizadas y que pueden ser una importante alternativa dentro de la proposición de estos sistemas?

\subsection{Fuentes de datos y estrategias de búsqueda}

Las bases de datos utilizadas para la búsqueda de proyectos de investigación fueron IEEEXplore ${ }^{\circledR}$, ACM, Springer, SciELO y Google ${ }^{\circledR}$ Académico. Para esto, se tuvo en cuenta la terminología referente a sistemas de información basados en AP. La tabla 1 muestra los patrones de búsqueda utilizados.

\subsection{Selección de estudios}

Para la selección de estudios se establecieron dos criterios para determinar la relevancia de los mismos: inclusión y exclusión. El primer término hace referencia a trabajos que se centran en sistemas de información y propuestas de arquitecturas para dichos sistemas; mientras que el segundo término hace referencia a los trabajos que no contengan los términos "sistema de información", "agricultura de precisión" y "sistema de soporte a decisiones". La tabla 1 muestra las cadenas de búsqueda utilizadas para seleccionar los trabajos relevantes dentro de la temática del presente estudio. 
Sistemas de información enfocados en tecnologías de agricultura de precisión y aplicables a la caña de azúcar... 111

Tabla 1. Cadenas de búsqueda y trabajos obtenidos

\begin{tabular}{|l|c|c|c|}
\hline \multicolumn{1}{|c|}{ Cadena de búsqueda } & $\begin{array}{c}\text { Número de } \\
\text { trabajos }\end{array}$ & $\begin{array}{c}\text { Número de trabajos } \\
\text { seleccionados }\end{array}$ & Precisión \\
\hline agricultural information system & $\sim 500$ & & $\sim 0 \%$ \\
\hline precision agriculture information system & $\sim 500$ & & $\sim 0 \%$ \\
\hline $\begin{array}{l}\text { agricultural information system AND sugarcane AND } \\
\text { unmanned aerial vehicles }\end{array}$ & 30 & 7 & $\sim 23 \%$ \\
\hline $\begin{array}{l}\text { precision agriculture information system AND sugar- } \\
\text { cane AND decision support systems }\end{array}$ & 20 & 12 & $\sim 60 \%$ \\
\hline precision agriculture AND Colombia & $\sim 80$ & 7 & $\sim 9 \%$ \\
\hline precision agriculture AND Colombia AND sugarcane & $\sim 7$ & 4 & $\sim 57 \%$ \\
\hline Total & & 30 & \\
\hline
\end{tabular}

Fuente: los autores

De los resultados en la tabla 1 se tiene que la cadena con la cual se obtuvieron los mejores resultados fue "precision agriculture information system AND sugarcane AND decision support systems", con un $60 \%$ de precisión. En esta etapa fueron seleccionados 30 trabajos relevantes para el presente estudio.

\subsection{Clasificación de artículos}

El proceso de clasificación de trabajos está basado en las tecnologías y procedimientos de AP que utilizan los autores de los artículos seleccionados en la sección anterior. La tabla 2 muestra los trabajos enfocados en los procesos de AP descritos en la sección 1 , junto con su peso porcentual.

Tabla 2. Clasificación de trabajos según tecnologías utilizadas.

\begin{tabular}{|l|c|c|}
\hline \multicolumn{1}{|c|}{ Tecnología o procedimiento de AP utilizado } & Número de trabajos & Porcentaje \\
\hline Sistemas de soporte a decisiones & 5 & $16.6 \%$ \\
\hline Aeronaves pilotadas remotamente & 5 & $16.6 \%$ \\
\hline Sensores remotos y arquitecturas de red & 5 & $16.6 \%$ \\
\hline Uso de sistemas de posicionamiento & 5 & $16.6 \%$ \\
\hline Sistemas integrales de información & 4 & $13.4 \%$ \\
\hline Sistemas de recomendación aplicados a cosechas & 3 & $10.1 \%$ \\
\hline Tecnologías de tasa variable & 3 & $10.1 \%$ \\
\hline
\end{tabular}

Fuente: los autores 


\subsection{Extracción de datos y síntesis}

Para obtener información detallada y concisa de cada trabajo previamente seleccionado y clasificado, se elaboró una plantilla de extracción de datos por cada estudio. En dicha plantilla se consignó la información principal de cada artículo: título, resumen, palabras clave, aportes, ventajas y falencias que derivan al final en las brechas relacionadas con el problema de investigación.

\section{TRABAJOS RELACIONADOS}

Dada la clasificación previamente realizada, los trabajos relacionados se clasificaron según lo muestra la tabla 2. A continuación se presenta una descripción general de los trabajos.

\subsection{Proyectos de investigación clasificados como DSS}

Cock et al. [10] presentan una metodología para determinar prácticas de gestión de cultivos mediante AP basadas en labores tradicionales. Los autores definen en su metodología la obtención de datos del cultivo que caracterizan las condiciones del mismo, la gestión y el análisis en bases de datos centralizadas y la facilidad en la accesibilidad de dicha información a los encargados del cultivo, para que tomen mejores decisiones relacionadas con el mismo. Demattê et al. [11] informan acerca de comparaciones entre procesos de muestreo, producción, daño en cultivo y costos al utilizar procedimientos y tecnologías de AP y al cultivar comúnmente caña de azúcar en Brasil, por lo que presentan recomendaciones y soporte a decisiones, basados en requerimientos puntuales del sitio de estudio. Por otra parte, López et al. [12] presentan resultados de desempeño de un sistema de recomendación de cinco fases para el uso de fertilizantes en cultivos de caña de azúcar, de donde indican los valores de nutrientes requeridos en cada dosis de fertilización aplicada. Stray et al. [13] proponen un DSS capaz de proveer apoyo computacional al personal encargado de programar operaciones de cosechado de caña de azúcar en Sudáfrica; el sistema propuesto es útil en situaciones donde el número de campos a monitorizar manualmente es alto y cuando las condiciones en las cuales crece el cultivo cambian frecuentemente.

\subsection{Trabajos relacionados con drones}

Estos elementos aprovechan las ventajas de vuelos a poca altura para la obtención de imágenes de alta resolución de cultivos, para la monitorización remota de los mismos y para la medición en aire de variables agroclimáticas, entre otros usos. Algunas de las investigaciones más recientes son las descritas en [14-17] las cuales evalúan de manera general las ventajas, desventajas, inconvenientes legales, aplicaciones actuales y potencial a futuro de su utilización en labores de AP. Para el caso de la obtención de 
imágenes de cultivos, se han definido varios índices de vegetación que son indicadores del desarrollo de cultivos y ayudan a predecir sus rendimientos futuros. Estos índices se calculan dada la notable diferencia de la reflectividad de las plantas entre la banda visible (radiación emitida en el proceso de fotosíntesis) y la infrarroja (radiación solar reflejada) del espectro electromagnético.

\subsection{Sensores remotos y arquitecturas de red}

Urbano [18] presenta el diseño preliminar de una WSN basada en el estándar IEEE 802.15.4, la cual presentó resultados iniciales de temperatura, humedad, radiación solar y flujo fotosintético sobre cultivos de café. El prototipo de red presenta una topología en estrella, con un nodo coordinador y tres nodos sensores, comunicados inalámbricamente con ayuda de módulos $\mathrm{Xbee}{ }^{\circledR}$. El autor recalca en que el consumo de energía de este tipo de sensores debe ser el mínimo posible para aumentar la vida útil de la red y prolongar la duración de las baterías. Sudduth et al. [19] presentan resultados de investigación acerca del uso de sensores de inducción electromagnética para la obtención de la conductividad eléctrica del suelo. Los autores presentan posibles errores de medición al momento de utilizar dichos sensores y la correspondiente salinidad, humedad, profundidad y arcilla del suelo estudiado, y proponen técnicas de calibración para la medición de estas variables en cultivos. Otros trabajos en redes de sensores son los realizados en [20-22], donde se presentan revisiones de las distintas variables agroclimáticas para medición en cultivos y maquinaria.

\subsection{Sistemas de recomendación aplicados a cosecha}

De Souza et al. [23] presenta un sistema de análisis de geoestadísticas para los atributos del suelo y del rendimiento en general de un cultivo de caña ubicado en Brasil. Los autores utilizan minería de datos (data mining) mediante inducción de árboles de decisión, obteniendo un mapa digital que representa la superficie de producción del área en estudio, además de obtener resultados acerca de cómo la altitud es la variable más representativa en relación al rendimiento del cultivo de caña. Por otra parte, Carbonell [24] informa acerca de los avances que el Centro de Investigación de la Caña de Azúcar de Colombia (Cenicaña) ha realizado en temas de AP, destacando la zonificación agroecológica realizada por esta entidad, la cual caracteriza los diversos tipos de suelo donde se encuentra plantado el cultivo en relación con los grupos de humedad y homogeneidad de los mismos.

\subsection{Tecnologías de tasa variable (VRT)}

Markley y Hughes [25] presentan un sistema para la aplicación de nitrógeno de manera automática y dependiendo de las necesidades del terreno. Estas necesidades son 
controladas por sensores de EC, junto con mapeo del terreno por medio de GPS; los resultados indican que altas variaciones de EC inciden en la productividad del cultivo. Serrano et al. [26] presentan un conjunto de desarrollos tecnológicos que optimizan la aplicación de fertilizantes mediante tractores; específicamente sobre sistemas de soporte a la conducción y gestión de la caja de cambios. Además, esta maquinaria está gestionada mediante GPS, y la aplicación de fertilizantes se basa en información de sensores ubicados en el cultivo.

\subsection{Sistemas de información}

Los trabajos encontrados que pueden clasificarse en esta sección son relativamente pocos, empezando por Ye et al. [27], quienes presentan resultados de investigación acerca de la aplicación del Internet de las Cosas (IoT: Internet of Things) y los SIG basados en web (WebGIS) en la agricultura. Proponen un modelo de gestión de AP basado en cuatro plataformas: plataforma de infraestructura de información espacial, plataforma de IoT, plataforma de gestión de agricultura y cliente móvil. El sistema fue aplicado a una granja ecológica, ayudando al personal a reducir el tiempo de monitorización de los cultivos al presentar información en tiempo real, mejorando así la rentabilidad de los mismos. La figura 2 muestra la arquitectura en detalle.

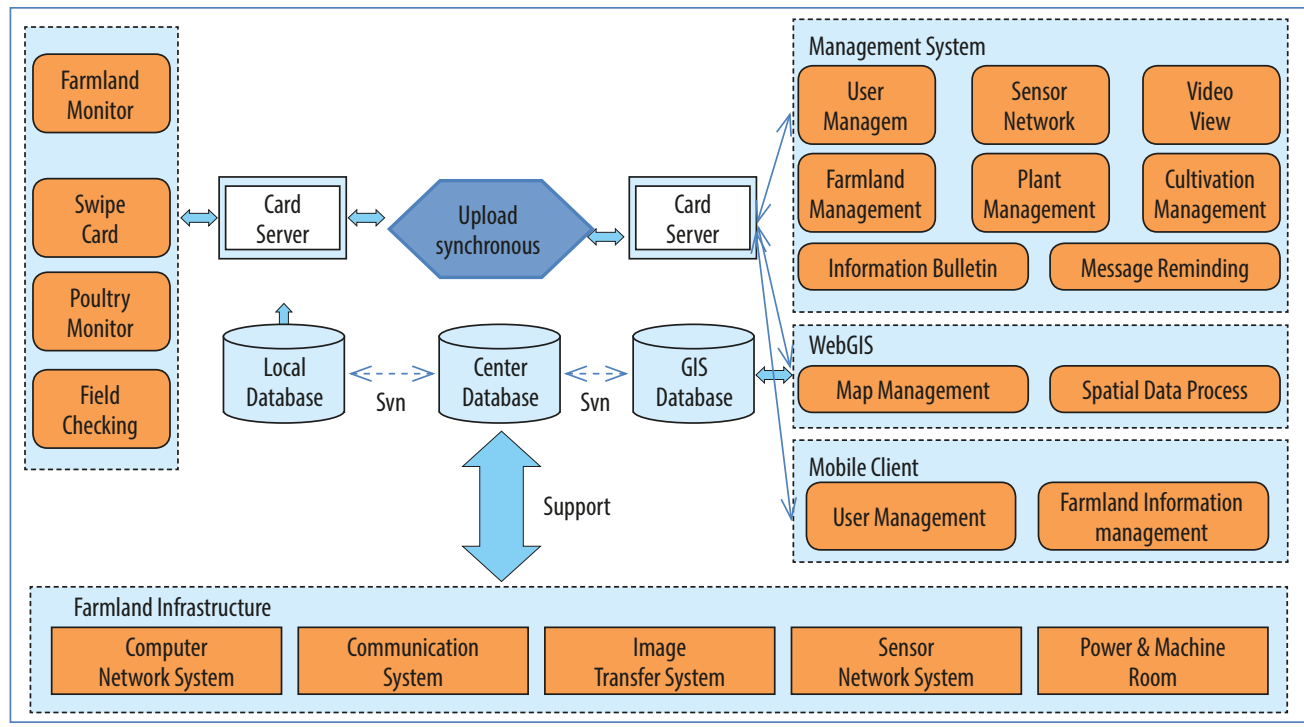

Figura 2. Arquitectura del sistema.

Fuente: [27]

Por otra parte, en [28], los autores analizan la evolución de diferentes elementos relacionados con cultivos, suelos, clima, personas, metas, objetivos y toma de decisiones, de donde se centran en seis tipos de análisis de sistemas agrícolas. Los resultados 
obtenidos son evaluados en dos escenarios diferentes con cultivos diferentes. La aproximación a un modelo de gestión, monitorización, simulación y comunicación se muestra en la figura 3 , en donde se observa un conjunto de procesos divididos en dos partes para facilitar la toma de decisiones, gestión y monitorización.
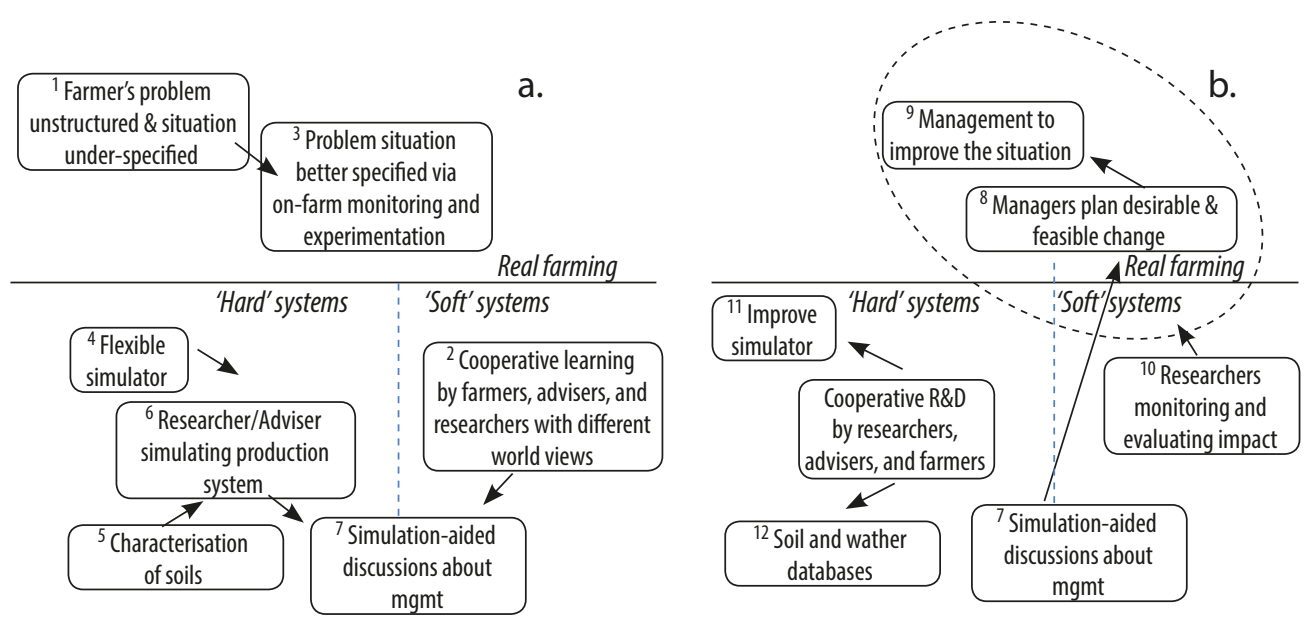

Figura 3. Modelo propuesto, (a) y (b) están unidos por el paso 7.

Fuente: [28]

En [29] se describe un análisis de datos para experimentos en AP, incluyendo aplicaciones para el caso de uso de la caña de azúcar. Este análisis se basa en la representación de técnicas de adquisición de datos, la estimación de ruido en cada medición, el análisis de autocorrelación espacial para cada atributo y el análisis de componentes principales (PCA: Principal Component Analysis) de los mismos. Los resultados obtenidos son datos de la diversidad del suelo y atributos del cultivo, medidos por técnicas de AP, obteniéndose una alta correlación en diversas variables. El sistema para dicho análisis se muestra en la figura 4, de donde se observa el tratamiento de información de manera correlacional y el análisis de PCA realizado. 


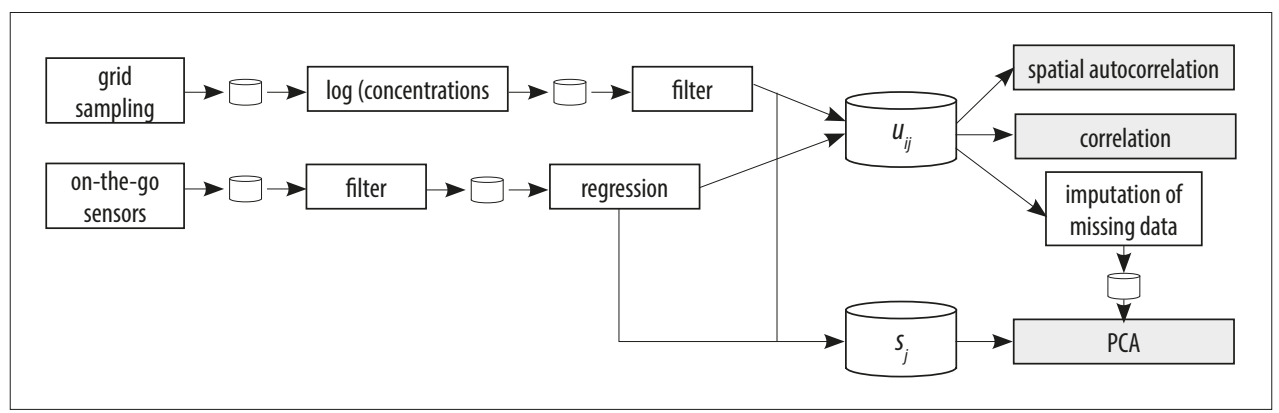

Figura 4. Flujo de análisis de datos.

Fuente: [29

Finalmente, Wang et al. [30] proponen un sistema de monitorización de información basado en arquitectura abierta, el cual consiste de redes de sensores junto con una plataforma de información del servicio. Los sensores capturan y reportan información entre ellos a través de técnicas multisalto y la transmiten mediante pasarelas GPRS hacia la plataforma de información, la cual genera reportes para informar a los encargados del cultivo. Además, los autores recalcan dos características que los sistemas de información deberían tener para optimizar sus aplicaciones: interfaces estándar y plataforma de servicio de información abierta. La figura 5 muestra la arquitectura del sistema propuesto por los autores.

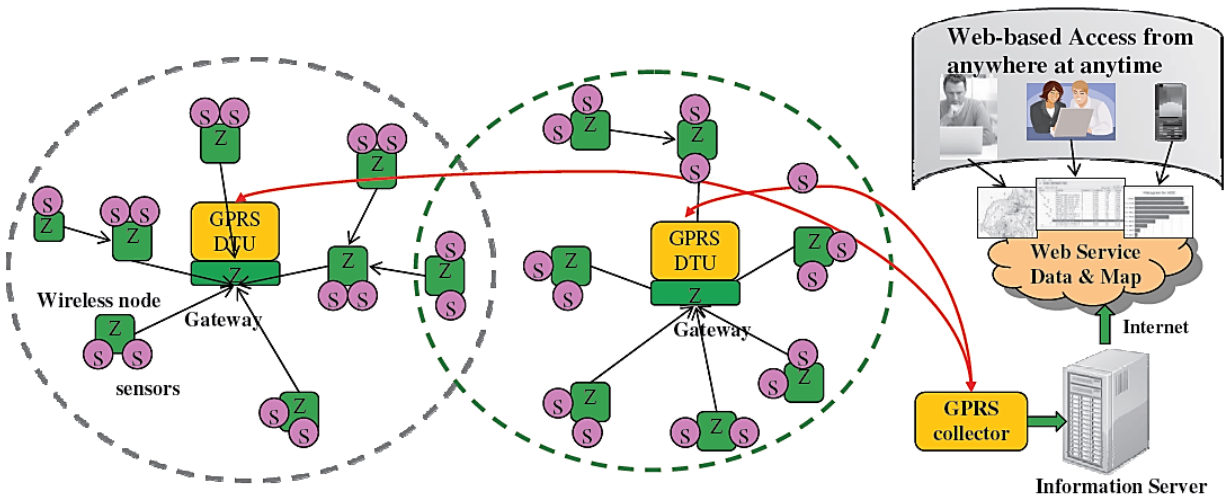

Figura 5. Arquitectura del sistema de monitorización.

Fuente: [30]

Con la revisión de literatura presentada anteriormente se observa que, aunque se encontraron proyectos de investigación relacionados con sistemas de información orientados hacia diferentes cultivos, estos se enfocan tanto en tecnologías como en etapas específicas del ciclo de vida, además de la resolución de necesidades particulares utilizando diferentes tecnologías y procedimientos. Por ende, son las aproximaciones 
más cercanas a la propuesta de este documento, la cual se diferencia de los anteriores trabajos al presentar una arquitectura integral, basada en software y hardware libres, apoyándose en RPA para la comunicación inalámbrica, enfocada en cultivos de caña orgánica y sus correspondientes prácticas, y capaz de informar al usuario final acerca de distintas variables agroclimáticas del cultivo en cuestión.

\subsection{Análisis del estudio de selección de artículos}

Aplicando los criterios de selección descritos en la sección 2.3, se obtuvo un conjunto de 30 trabajos relacionados con el tema de investigación. Como resultado de la revisión de literatura se presentan las respuestas a las preguntas de investigación de la sección 2.1.

Teniendo en cuenta la pregunta inicial de investigación, referente a temas de interés para la comunidad científica dentro de una ventana de tiempo reciente, los sistemas de información representan un amplio campo de investigación en el cual los autores enfocan sus trabajos alrededor de subtemas específicos. Dichos subtópicos son principalmente las tecnologías descritas en la sección 1, como los trabajos con RPA, basados en redes de sensores y DSS.

Para responder a la segunda pregunta de investigación acerca de las características que presentan estos sistemas en relación con su arquitectura, funcionamiento y aplicabilidad, los trabajos encontrados describen la arquitectura y su funcionamiento de manera general. Es así como los sistemas de información descritos en [27-30] describen gráficamente su arquitectura y operación, presentando características comunes como: utilización de sensores para la captura de datos en terreno, presentación de datos a las personas encargadas de los cultivos, transmisión inalámbrica de datos, entre otras, por lo que estas características comunes encontradas en los sistemas descritos por los respectivos autores serán tenidas en cuenta para la propuesta presentada en este artículo.

Para dar respuesta a la última pregunta de investigación acerca de técnicas y procedimientos no explorados en la proposición de sistemas de información basados en AP, la investigación realizada encontró brechas al momento de la implementación de sistemas integrales de información multifuncionales, por lo que la propuesta presentada planea la integración de varias tecnologías de agricultura de precisión en un sistema integral. Tecnologías como redes de sensores integradas con RPA, además de DSS y monitorización por GPS están proyectadas a ser implementadas en la propuesta. También, el sistema propuesto estará en capacidad de medir, procesar y presentar información de gases de efecto invernadero (GEI) de los distintos procesos del cultivo para caracterizar la huella de carbono de la caña de azúcar. 


\section{SISTEMA PROPUESTO}

De acuerdo con estudios previos de organizaciones relacionadas con la industria azucarera colombiana, las compañías azucareras requieren nuevas tecnologías, modelos, sistemas y elementos para mejorar el proceso de cultivo de caña de azúcar. En consecuencia, nace esta propuesta de un sistema integral de información basado en AP para el cultivo de dicho producto en Colombia y con posibilidad de ser adaptado a otros cultivos.

\subsection{Arquitectura}

El sistema propuesto tendrá en cuenta tres dominios de arquitectura, con el fin de obtener una completa realimentación durante el ciclo de vida del producto y presentar resultados confiables y aplicables a los cultivos de caña del país. En la figura 6 se muestra la arquitectura propuesta.

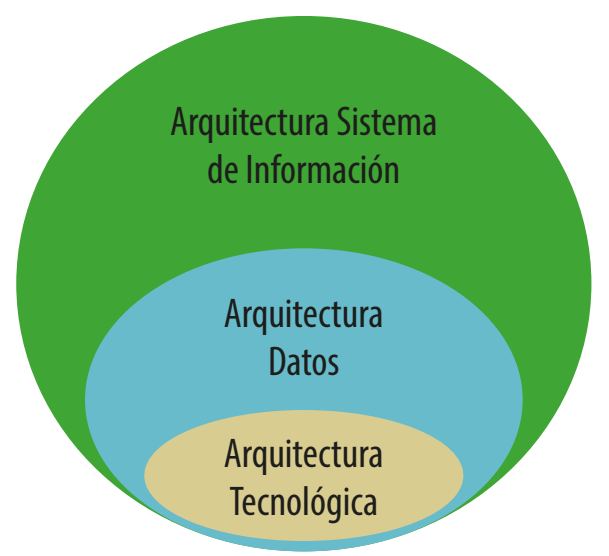

Figura 6. Arquitectura general del sistema propuesto.

Fuente: los autores

- Arquitectura tecnológica: define los componentes y elementos tecnológicos del sistema. Está constituida por los equipos para procesamiento, almacenamiento y redes de datos; la arquitectura de los sensores, sus tecnologías de comunicación y los medios de comunicación requeridos para la recolección de datos. Este dominio define los siguientes elementos:

- Requerimiento de los datos entregados por los SIG y GPS.

- Arquitectura hardware de los medios de procesamiento y almacenamiento de los datos. 
- Arquitectura técnica de los sensores y sus tecnologías de comunicación inalámbrica.

- Arquitectura de datos: precisa la estrategia para la organización, integración y consolidación de los datos obtenidos por medio de la arquitectura tecnológica. Permite la generación de informes, indicadores y tableros de control, lo que facilita la toma de decisiones de tipo técnico y económico, al facilitar mayores y mejores elementos de juicio. Este dominio define entre sus principales características cómo será:

- El flujo de datos obtenidos de los SIG, GPS y su integración con las TIC.

- La estructura, características y niveles de almacenamiento de los datos.

- Ciclo de vida, calidad y perdurabilidad de los datos obtenidos.

- Tecnología de intercambio de datos entre distintos repositorios.

- Arquitectura del sistema de información: detalla la estrategia para la organización de los sistemas de información empresarial, garantizando su correcta alineación con los procesos del negocio. Además, incorpora las soluciones aplicativas que apoyan el sistema propuesto e identifica los componentes y servicios que den respuesta a necesidades comunes de las áreas de negocio. Define principalmente los siguientes elementos:

- Integración del sistema de información con los datos generados en la arquitectura tecnológica.

- Generación de reportes para la toma de decisiones por el área o unidad de negocio responsable.

Es preciso insistir que el sistema propuesto busca la optimización del proceso de cultivo de la caña de azúcar orgánica en Colombia. Por ende, la aplicación de tecnologías de AP que se busca está enfocada a todas las etapas del ciclo de vida del cultivo (ver figura 7). Asimismo, se trabajará en asocio con una hacienda poseedora de cultivos de caña de este tipo, por lo que los requerimientos y necesidades que presenten serán los retroalimentados al sistema para buscar obtener los resultados esperados, mostrados más adelante. 


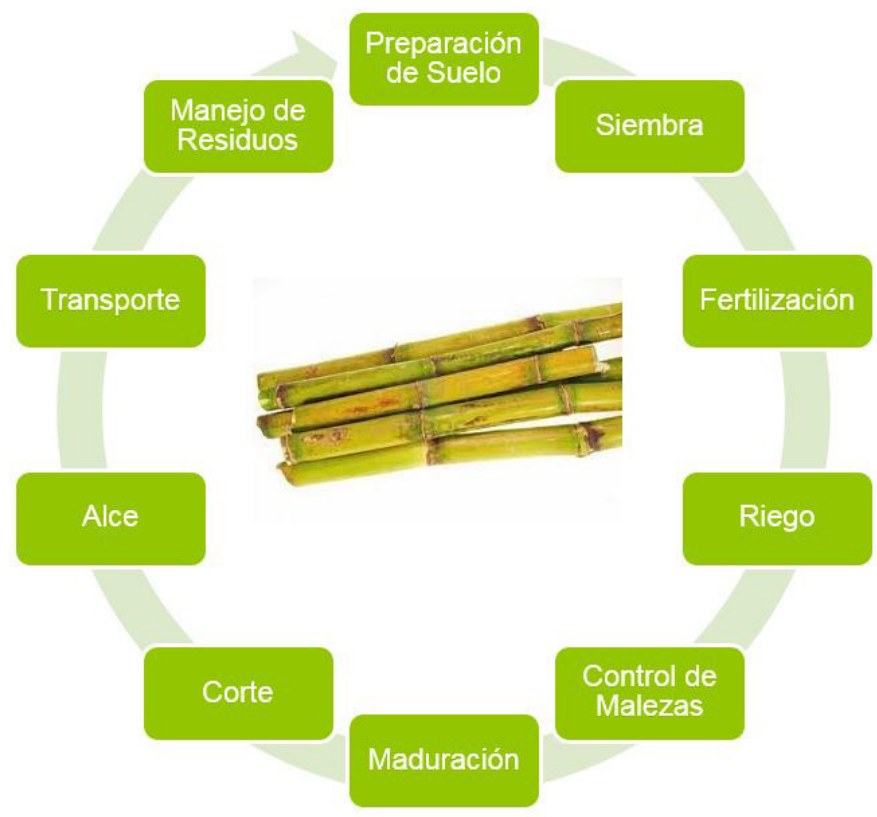

Figura 7. Etapas del ciclo de vida del cultivo de caña de azúcar. Fuente: los autores

\subsection{Motivación}

La necesidad de optimizar el proceso de cultivo de la caña de azúcar en Colombia, los requerimientos que las compañías azucareras presentan actualmente basados en capacidades de procesamiento, clima, tipo de suelo, etc., y la necesidad de disminuir la huella de carbono producida por la agricultura, particularmente por el ciclo de vida de la caña de azúcar hacen que la propuesta presentada en este documento esté alineada a proponer soluciones en estos tópicos. Los actores involucrados en el cultivo de caña de azúcar requieren herramientas eficientes, confiables y robustas para mejorar sus prácticas empresariales, por lo que la propuesta descrita busca satisfacer dichos requerimientos. El sistema descrito involucrará requerimientos generales en el cultivo de caña de azúcar orgánica, pero será escalable y adaptable a otro tipo de caña y cultivo.

\subsection{Resultados esperados}

Los entregables y resultados deseados para ser presentados por el sistema propuesto son los siguientes:

- Mapas de productividad: monitores de productividad en maquinaria agrícola, optimización de rutas. 
- Mapas de fertilidad del suelo: determinación de niveles de nutrientes para análisis de suelos, adecuada aplicación dependiendo de necesidades, clima, época del año, etc.

- Mapas de recomendación: fertilización ideal, mapas de aplicación, trazabilidad de procesos de aplicación.

- Mapas de emisiones de GEI: información detallada de GEI emitidos, recomendaciones para su reducción.

\subsection{Trabajo futuro}

La tarea principal a futuro será implementar cada uno de los dominios mostrados en la figura 6, siguiendo los lineamientos de la investigación paralela sobre GEI que realizará el grupo ICUBO acerca de huella de carbono; conjuntamente se tendrán en cuenta los requerimientos puntuales de la hacienda donde se realizarán pruebas de campo. Se analizarán los procedimientos realizados durante el cultivo del producto, con el fin de sintetizar soluciones en el marco de AP que optimicen el uso de recursos como agua y fertilizantes, además de implementar el sistema de monitorización y sensado remoto de características del suelo.

\section{CONCLUSIONES}

La agricultura de precisión se presenta como una técnica novedosa para aumentar la productividad de las empresas agrícolas. Busca optimizar el proceso de cultivo de un producto mediante el uso de GPS, SIG, sensores, drones, etc., y, aunque presenta desafíos para su implementación, se considera como prometedora para afrontar las crecientes necesidades de la industria agrícola.

Este artículo presentó una revisión de la literatura enfocada en sistemas de información para AP, encontrándose que, si bien la investigación en esta área es considerable, pocos proyectos se han enfocado en su descripción. Por consiguiente, surge la propuesta de un sistema de información basado en AP, compuesto de tres (3) dominios que abarcan todo el ciclo de vida del cultivo de caña de azúcar. Las características, enfoques y posibles resultados a obtener están definidos por las necesidades generales de los ingenios azucareros colombianos, del tipo de suelo, del clima de la región, entre otros.

La investigación continuará definiendo específicamente los componentes de cada dominio, junto con visitas técnicas a la hacienda seleccionada para observar las etapas del ciclo de vida del cultivo y proponer, mediante análisis de datos, estrategias para la implementación de AP. 


\section{AGRADECIMIENTOS}

Los autores de este artículo agradecen el apoyo financiero recibido por el Departamento Administrativo de Ciencia, Tecnología e Innovación (Colciencias) y su programa de Jóvenes Investigadores en el marco del convenio 0194 de 2014 y de la convocatoria 645 de 2014; además se agradecen las sugerencias y valiosos comentarios brindados por los miembros del grupo de investigación ICUBO y del grupo de investigación en informática y telecomunicaciones (i2t) de la Universidad Icesi.

\section{REFERENCIAS}

[1] Food and Agriculture Organization for the United Nations, "FAO Statistical Yearbook 2013: World food and agriculture," Roma, 2013.

[2] C. Silva, M. Moraes, y J. Molin, "Adoption and use of precision agriculture technologies in the sugarcane industry of São Paulo state, Brazil," Precision Agriculture, vol. 12, n. ${ }^{\circ}$ 1, pp. 67-81, 2010.

[3] R. Bramley, "Lessons from nearly 20 years of Precision Agriculture research, development, and adoption as a guide to its appropriate application," Crop. Pasture Science, vol. 60, n. ${ }^{\circ}$, pp. 197-217, 2009.

[4] R. Plumb, "Precision agriculture in the 21st century: geospatial and information technologies in crop management," Pest Management Science, vol. 56, n. ${ }^{\circ}$ 8, pp. 723-723, 2000.

[5] R. Grisso, M. Alley, P. McClellan, D. Brann, y S. Donohue, "Precision Farming. A Comprehensive Approach," Virginia Cooperative Extension, Publication 442-500, Virginia State University, 2009.

[6] A. Dobermann, S. Blackmore, S. Cook, y V. Adamchuk, "Precision Farming: Challenges and Future Directions," presentado en Proceedings of the 4th International Crop Science Congress, Brisbane, Australia, 2004.

[7] P. Tozer, "Uncertainty and investment in precision agriculture - Is it worth the money?" Agricultural Systems, vol. 100, n. ${ }^{\circ} 1-3$, pp. 80-87, 2009.

[8] M. Rilwani y J. Oghenereemusua, "Geoinformatics in Agricultural Development: Challenges and Prospects in Nigeria," Journal of Social Sciences, vol. 21, n. ${ }^{\circ}$ 1, pp. 49-57, 2009.

[9] B. Kitchenham y S. Charters, "Guidelines for performing Systematic Literature Reviews in Software Engineering," Keele University and Durham University Joint Report, UK, EBSE 2007-001, 2007.

[10] J. Cock et al., "Crop management based on field observations: Case studies in sugarcane and coffee," Agricultural Systems, vol. 104, n. ${ }^{\circ}$ 9, pp. 755-769, 2011.

[11] J. Demattê, L. Demattê, E. Alves, R. Negrão, y J. L. Morelli, "Precision agriculture for sugarcane management: a strategy applied for brazilian conditions," Acta Scientiarum. Agronomy, 
vol. 36, n. ${ }^{\circ}$ 1, pp. 111-117, 2014.

[12] D. López et al., "Sistema integrado para recomendar dosis de fertilización en caña de azúcar (SIRDF),” Terra Latinoamericana, vol. 20, n. ${ }^{\circ}$ 3, pp. 347-358, 2002.

[13] B. Stray, J. van Vuuren, y C. Bezuidenhout, "An optimisation-based seasonal sugarcane harvest scheduling decision support system for commercial growers in South Africa," Computers and Electronics in Agriculture, vol. 83, pp. 21-31, 2012.

[14] C. Zhang, D. Walters, y J. M. Kovacs, "Applications of Low Altitude Remote Sensing in Agriculture upon Farmers' Requests - A Case Study in Northeastern Ontario, Canada,” PLoS ONE, vol. 9, n. ${ }^{\circ}$ 11, pp. 1-9, 2014.

[15] G. López, "Diseño de un programa de ortorectificación y georreferenciación de imágenes aéreas aplicadas a campos de caña de azúcar,” Pontificia Universidad Católica del Perú, Lima, Perú, 2014.

[16] G. Schneider, A. Hadad, y A. Kemerer, "Implementación de un software para el análisis de imágenes aéreas multiespectrales de caña de azúcar,” Ventana Informática, vol. 28, n. 1, pp. 13-29, 2013.

[17] E. Hunt, C. Daughtry, S. Mirsky, y W. Hively, "Remote Sensing With Simulated Unmanned Aircraft Imagery for Precision Agriculture Applications," IEEE Journal of Selected Topics in Applied Earth Observations and Remote Sensing, vol. 7, n. ${ }^{\circ}$ 11, pp. 4566-4571, 2014.

[18] F. Urbano, "Redes de sensores inalámbricos aplicadas a optimización en agricultura de precisión para cultivos de café en Colombia,” Journal de Ciencia e Ingeniería, vol. 5, n. 1, pp. 46-52, 2013.

[19] K. Sudduth, S. Drummond, y N. Kitchen, "Accuracy issues in electromagnetic induction sensing of soil electrical conductivity for precision agriculture," Computers and Electronics in Agriculture, vol. 31, n. 3 , pp. 239-264, 2001.

[20] N. Nawi, G. Chen, y T. Jensen, "In-field measurement and sampling technologies for monitoring quality in the sugarcane industry: a review," Precision Agriculture, vol. 15, n. ${ }^{\circ}$, pp. 684-703, 2014.

[21] R. Price, R. Johnson, R. Viator, J. Larsen, y A. Peters, "Fiber Optic Yield Monitor for a Sugarcane Harvester," Transactions of the ASABE, vol. 54, n. ${ }^{\circ}$, pp. 31-39, 2011.

[22] J. Molin, F. Frasson, L. Amaral, F. Povh, y J. Salvi, "Capability of an optical sensor in verifying the sugarcane response to nitrogen rates," Revista Brasileira de Engenharia Agrícola e Ambiental, vol. 14, n. ${ }^{\circ}$ 12, pp. 1345-1349, 2010.

[23] Z. de Souza et al., "Analyze the soil attributes and sugarcane yield culture with the use of geostatistics and decision trees," Ciência Rural, vol. 40, n. ${ }^{\circ} 4$, pp. 840-847, 2010.

[24] J. Carbonell, "Experiencia del sector cañicultor en agricultura específica por sitio," Palmas, vol. 29, n. $^{\circ} 2$, pp. $65-70,2008$. 
[25] J. Markley y J. Hughes, "Understanding the Barriers to the Implementation of Precision Agriculture in the Central Region," presentado en 35th Annual Conference of the Australian Society of Sugar Cane Technologists, Townsville, Australia, 2013.

[26] J. Serrano, J. Peça, J. Silva, y S. Shahidian, “Aplicação de fertilizantes: tecnologia, eficiência energética e ambiente”. Revista de Ciências Agrárias, vol. 37, n. 3, pp. 270-279, 2014.

[27] J. Ye, B. Chen, Q. Liu, y Y. Fang, “A precision agriculture management system based on Internet of Things and WebGIS," presentado en 2013 21st International Conference on Geoinformatics (GEOINFORMATICS), Kaifeng, China, 2013.

[28] B. Keating y R. McCown, "Advances in farming systems analysis and intervention," Agricultural Systems, vol. 70, n. ${ }^{\circ} 2-3$, pp. 555-579, 2001.

[29] C. Driemeier et al., "Data Analysis Workflow for Experiments in Sugarcane Precision Agriculture," in 2014 IEEE 10th International Conference on e-Science (e-Science), Guarujá, Brasil, 2014.

[30] Y. Wang, Y. Wang, X. Qi, y L. Xu, “OPAIMS: open architecture precision agriculture information monitoring system," presentado en Proceedings of the 2009 International conference on Compilers, architecture, and synthesis for embedded systems, Grenoble, Francia, 2009. 